\title{
An Enhanced Virtual Space Vector Modulation Scheme of Three-Level NPC Converters for More-Electric-Aircraft Applications
}

\author{
Feng Guo, Student Member, IEEE, Tao Yang, Senior Member, IEEE, Ahmed M. Diab, Student Member, IEEE, \\ Seang Shen Yeoh, Serhiy Bozhko, Senior Member, IEEE, and Patrick Wheeler, Fellow, IEEE
}

\begin{abstract}
Multilevel converters have been used for aircraft electric starter/generator (ESG) systems due to their high power qualities. One of the desirable topologies is the three-level neutralpoint-clamped (3L-NPC) converter. Our studied ESG system operates at a high speed during its generation mode, which results in high modulation index (MI) and puts some specific challenges on neutral-point (NP) voltage balance, especially under low power factor (PF) load conditions. Moreover, common-mode voltage (CMV) needs to be addressed properly as it leads to irreversible damage of motor shaft bearings, thereby degrading the efficiency and reliability of the entire system. Compared with the conventional nearest-three virtual space vector $\left(\mathrm{NTV}^{2}\right)$ technique, the proposed modulation scheme employs three adjacent medium vectors to synthesize a new medium vector and two pairs of large vectors to compose new small vectors. This allows the presented modulation scheme to achieve balanced capacitor voltage and reduced CMV at the same time. In addition, the torque ripple of the proposed modulation strategy is thoroughly compared and analyzed. Meanwhile, in order to overcome the heavy computational burden, a fast calculation approach is adopted to simplify the algorithm. Simulation results obtained from Simulink/PLECS and experimental results obtained from a 45 $\mathrm{kW}, 32 \mathrm{krpm}$ aircraft ESG system verify the effectiveness of the proposed strategy in more-electric-aircraft (MEA) applications.
\end{abstract}

Index Terms - Common-mode voltage, low-complexity, neutralpoint voltage balance, three-level, virtual space vector.

\section{INTRODUCTION}

$\mathrm{T}$ HE more-electric-aircraft (MEA) brings many potential benefits in the design and carbon emissions of future civil aircraft for the aerospace industry [1]-[5]. A key technology of the MEA concept is the ESG system that serves as the interface for delivering electrical energy to onboard electrical power system (EPS). To achieve high efficiency of fuel consumption in aircraft, the ESG system shown in Fig.1 mainly consists of a permanent magnet synchronous machine (PMSM) and a bidirectional AC-DC converter. The PMSM is mechanically

This work was supported by the Clean Sky 2 Joint Undertaking under Grant 807081. (Corresponding author: Tao Yang.) The author Feng Guo would like to thank China Scholarship Council (CSC) for sponsoring part of his Ph.D studentship.

F. Guo, T. Yang, S. S. Yeoh, S. Bozhko and P. Wheeler are with the Power Electronics, Machines and Control Group, the Department of Electrical and Electronic Engineering, The University of Nottingham, Nottingham NG7 2RD, U.K. (email: feng.guo@nottingham.ac.uk; tao.yang@nottingham.ac.uk; seang.yeoh@nottingham.ac.uk; pat.wheeler@nottingham.ac.uk) serhiy.bozhko@nottingham.ac.uk;

Ahmed M. Diab is with the Key Laboratory of More Electric Aircraft Technology of Zhejiang Province, University of Nottingham Ningbo China, Ningbo 315100, China (e-mail: ahmed.diab@nottingham.edu.cn)

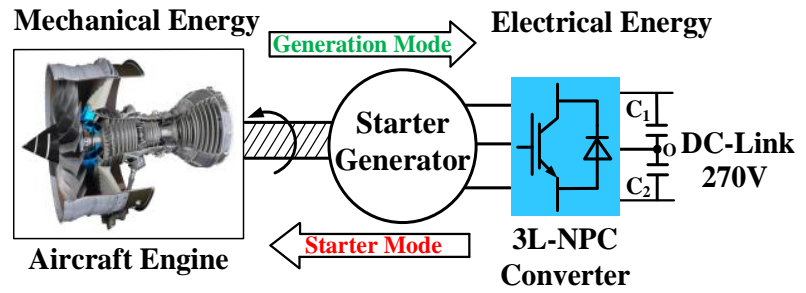

Fig.1. ESG system of more-electric-aircraft.

coupled to the aircraft engine shaft and electrically connected with a bidirectional converter. Compared with two-level counterpart, 3L-NPC multilevel converter is preferable due to its mature topology with lower voltage stress on power devices and better output harmonics. In terms of other multilevel topologies, additional isolated DC sources are required for the cascaded H-bridge (CHB) inverters [6], and bulky capacitors are necessary for the flying-capacitor (FC) inverters [7]. Considering the stringent requirement of weight and volume in the aerospace industry, the 3L-NPC converter with a single DC source shown in Fig. 2 is selected and discussed in this work.

In the beginning, the converter runs as an inverter that makes PMSM crank aircraft engine. Once the machine speed approaches $10 \mathrm{krpm}$, the engine ignites at this moment. After that, the ESG system is in standby mode. When the engine speed accelerates to $20 \mathrm{krpm}$, the generation mode starts. In this mode, the engine cranks the PMSM that works as a generator and $3 \mathrm{~L}-\mathrm{NPC}$ converter operates as a rectifier to convert $\mathrm{AC}$ power into DC for supplying onboard loads.

It can be seen that $3 \mathrm{~L}-\mathrm{NPC}$ converter plays a significant role in the efficiency and reliability of the whole systems. Nevertheless, an inherent problem of this topology is the NP voltage imbalance due to the split DC-link capacitors. The potential drift in the NP causes extra over-stress on switching devices. Besides, it produces low-order harmonics on the motor side. The traditional modulation method reported in [8] predominantly applies small vectors. To manipulate NP currents generated by medium vectors, the nearest-three space vectors (NTV) involving P- or N-type small vector are chosen in a switching period. In [9], according to NP unbalanced range, the authors propose a feedback control algorithm to dynamically allocate duty cycle of each small vector. To achieve average zero NP current, the concept of nearest-three virtual space vector $\left(\mathrm{NTV}^{2}\right)$ modulation scheme is firstly presented in [10], which is capable of balancing capacitor voltages in full range of modulation indices and power factors. In the work of [11], an active modulation scheme has been studied for high-speed drives, which explores a generic bias- 


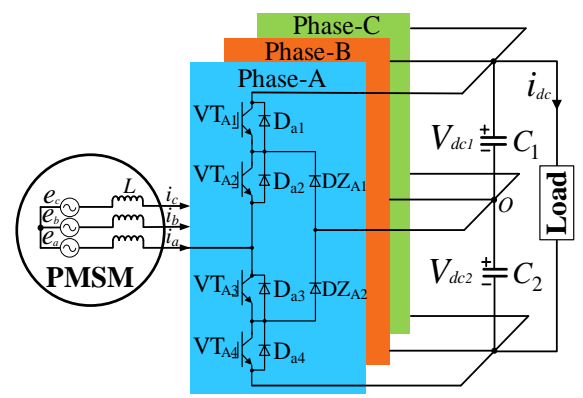

Fig.2 Topology of 3L-NPC converter.

offset injection method to settle this issue. Moreover, the NTV² based schemes have been developed for optimizing more objectives [12]-[14]. Considering the high modulation index and low power factor operating conditions of the designed ESG system [10], the virtual space vector modulation technique becomes a desirable candidate to solve the drawback.

On the other hand, motor drive systems fed by three-phase pulse-width-modulation (PWM) converters have the following disadvantages caused by the CMV:

1) High-frequency leakage current flowing to the ground through motor stray capacitor [15].

2) Winding insulation problem caused by bearing current due to motor shaft voltage [16].

3) Radiated electromagnetic interface (EMI) [17].

In terms of hardware-intensive approaches, common-mode (CM) choke is generally applied to reduce ground leakage current [18]. Machine winding insolation issues can be alleviated by adopting insulated bearing [15]. EMI can be suppressed by CM filters [19]. However, bulky size and extra cost should be carefully considered for aerospace applications. By contrast, new topology and advanced modulation strategy are two main solutions that can tackle the problem from the source. A balanced inverter topology has been proposed to cancel CMV in variable-speed motor drives [20].

From the PWM algorithm perspective, a virtual space vector modulation method for reducing CMV has been studied in [21], but it can be only applicable for two-level inverter applications. As medium and zero vectors do not generate CMV, reference voltage vector can be synthesized by these vectors to attain zero CMV [22]. Nevertheless, the restriction on modulation index makes it hard to be employed in some fields that require a higher DC-link voltage utilization. By using six virtual medium vectors, the shortcoming of this method has been fixed in [23]. However, because of the participation of medium vector, NP voltage oscillation becomes severe. Thus, large DC-link capacitance is needed. In [24], the authors present a modulation method for a three-level T-type inverter based on model predictive control (MPC) to gain balanced NP voltage and lower CMV. A modified NTV²-based modulation scheme applied in a variable-speed drive system is presented to keep capacitor voltage balanced and suppress CMV within one-third of DC-link voltage [25]. Since only [OOO] is chosen in the predefined switching sequence, CMV generated by the other two zero switching states can be avoided. However, it is worth noting that the method may cause two more switches turned on/off in one phase leg. In [26], a method adopting four vectors

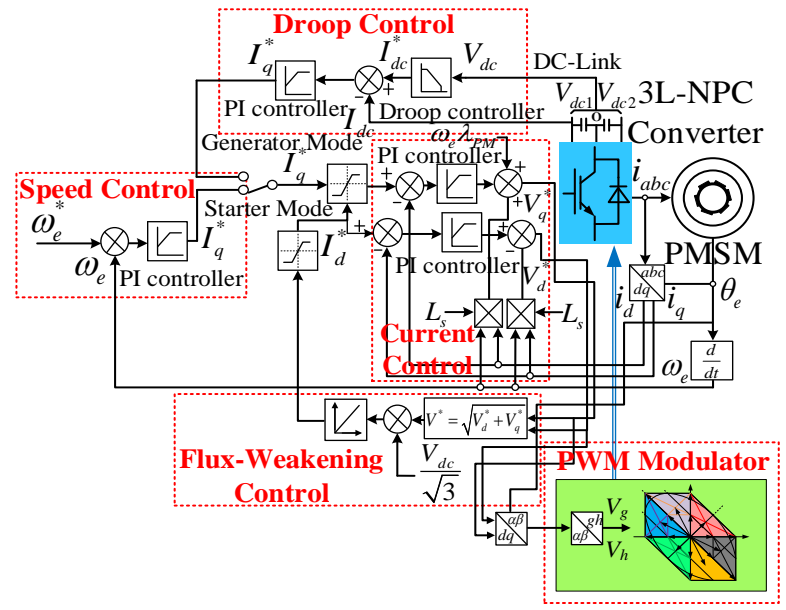

Fig. 3. Control blocks of designed ESG system.

with less CMV is investigated, together with a modified sector repartition. While it should point out that adjustment of dwell time of small vector is ineffective in weak NP voltage balance region. In [27], by defining two pairs of virtual small vectors with lower CMV, an advanced $\mathrm{NTV}^{2}$ strategy is proposed for eliminating NP voltage oscillation and mitigating CMVs. However, this algorithm is complicated as the switching sequence varies in each subsector. References [28] and [29] investigate carrier-based PWM (CBPWM) schemes to reduce CMV and balance NP voltage for a three-level back-to-back converter. It is noticeable that, through arranging switching sequences, the CMV can be limited as a single pulse during a switching period [29].

To attain balanced NP voltage and mitigated CMV for the studied ESG system, a modified $\mathrm{NTV}^{2}$ strategy is presented in this paper. Besides, for the sake of high computational efficiency, the fast calculation approach is adopted for simplifying sector identification and dwell time determination of the proposed PWM algorithm. The introduction and preliminary results of this work have been presented in [30].

The remaining part of this paper is structured as follows. In Section II, the principle of the ESG system is introduced. In Section III, the NTV and NTV ${ }^{2}$ schemes are briefly reviewed. Section IV analyzes the CMV of these two modulations and presents details of the proposed modulation strategy. In addition, the torque ripple and power losses are investigated and compared with the conventional $\mathrm{NTV}^{2}$ scheme. The simulation and experimental results are provided in Section V. Section VI summarize the main conclusions of this work.

\section{PRINCIPLES OF ESG SySTEM}

\section{A. Characteristics and Control of ESG System}

In our designed ESG system, the PMSM is chosen due to its high torque-to-current ratio, high power density and fault tolerance capability [31]. Although higher efficiency can be attained by increasing pole numbers, the low pulse-ratio problem would be expected due to high fundamental frequency [32]. Based on these reasons, 6-poles 36-slots surface-mounted PMSM is selected as the optimal candidate for the design and manufacturing [33]. 


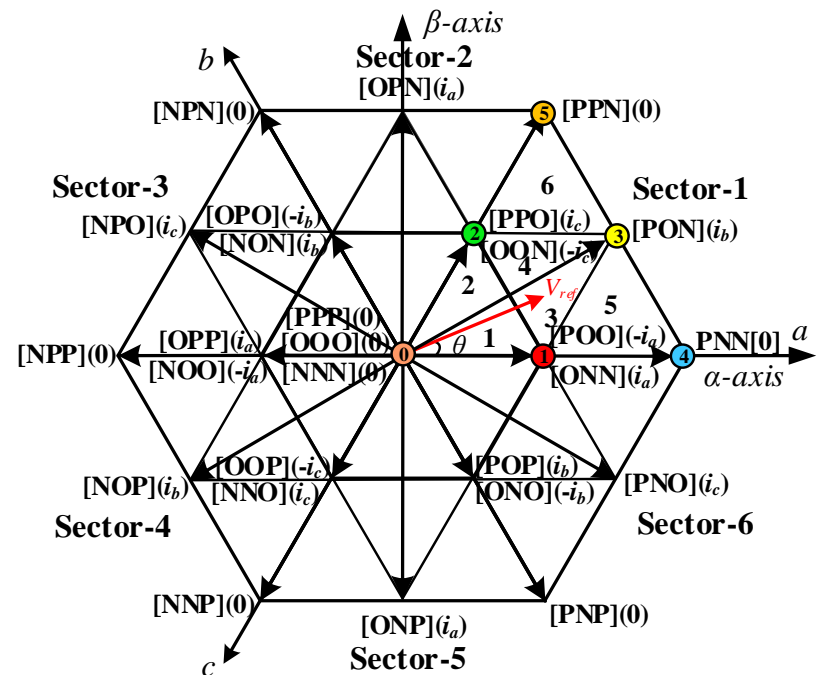

Fig.4. SVD of NTV scheme for 3L-NPC converter.

TABLE I

THE SWITCHING PRINCIPLES OF 3L-NPC CONVERTER

\begin{tabular}{cccccc}
\hline \hline Switching & \multicolumn{3}{c}{ SwITCH STATUS } & Output Voltage \\
State & $\mathrm{VT}_{x 1}$ & $\mathrm{VT}_{x 2}$ & $\mathrm{VT}_{x 3}$ & $\mathrm{VT}_{x 4}$ & Level \\
\hline $\mathrm{P}$ & 1 & 1 & 0 & 0 & $V_{d c} / 2$ \\
$\mathrm{O}$ & 0 & 1 & 1 & 0 & 0 \\
$\mathrm{~N}$ & 0 & 0 & 1 & 1 & $-V_{d c} / 2$ \\
\hline \hline
\end{tabular}

The ESG control blocks are illustrated in Fig.3. As shown, the outer control loop consists of flux-weakening control for high-speed operation, machine speed control for starter mode and DC-link voltage control for generation mode. The inner control loop is designed for current control. Considering the robustness issue, the magnitude of space voltage vector is still calculated based on the classical current PI control. The vector control technique is used for machine decoupling purposes, thereby linearizing PMSM mathematical model, which introduces flux and torque independently control. Therefore, machine torque represented as active power can be controlled by $q$-axis current. As large back electromotive force (EMF) is required to handle when the machine speed runs above $14 \mathrm{krpm}$, a large negative $d$-axis current must be injected to make PMSM operate in the flex-weakening region, which results in a low power factor. Speed control is executed in the startup process, and DC-link current is controlled according to the droop characteristics under power generating conditions.

\section{B. Three-Level NPC Converter}

As shown in Fig.2, each phase of the 3L-NPC converter includes four IGBTs $\left(\mathrm{VT}_{x 1} \sim \mathrm{VT}_{x 4}\right)$ and two clamping diodes $\left(\mathrm{DZ}_{x 1}\right.$ and $\mathrm{DZ} \mathrm{Z}_{x 2}$ ), where $x$ denotes phase $\mathrm{A}, \mathrm{B}$ and $\mathrm{C}$. Two equal capacitors $\left(C_{1}\right.$ and $\left.C_{2}\right)$ are connected in series. The topology clamps each phase voltage to the NP and positive/negative rail of the DC-link. Thus, it produces three-level output voltages $\left( \pm V_{d c} / 2\right.$ and 0$)$. The relationship among switching state, switch status and output voltage level is detailed in Table I.

\section{NTV AND NTV² SCHEMES FOR 3L-NPC CONVERTER}

\section{A. NTV}

The topology of 3L-NPC converter offers 27 combinations of switching state that correspond to different types of space

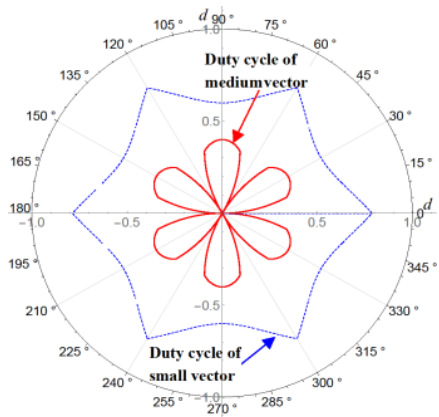

(a)

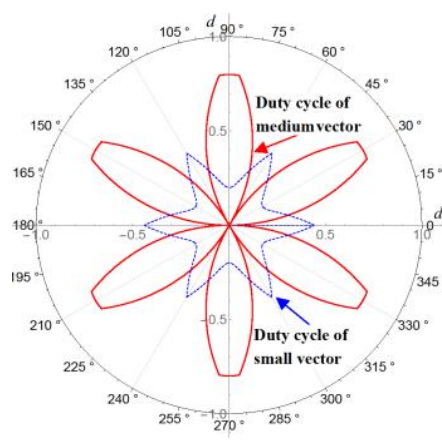

(c)

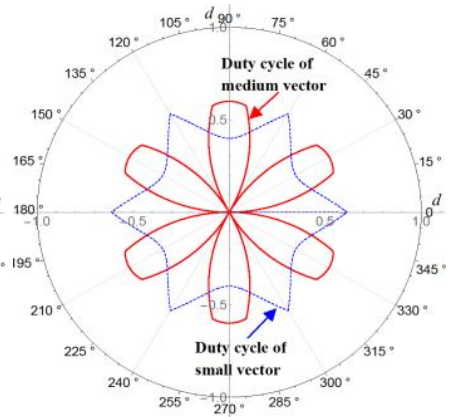

(b)

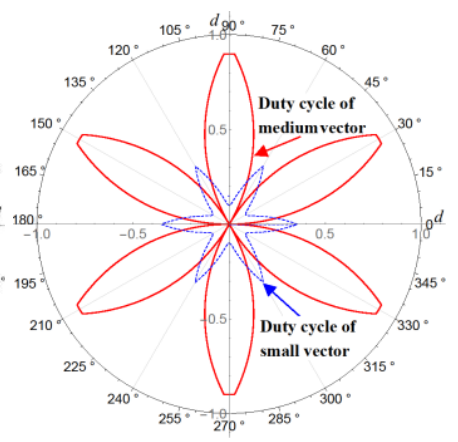

(d)
Fig.5. Duty cycle polar plot of small and medium vector with (a) MI=0.7. (b) $\mathrm{MI}=0.8$. (c) $\mathrm{MI}=0.9$. (d) $\mathrm{MI}=0.95$.

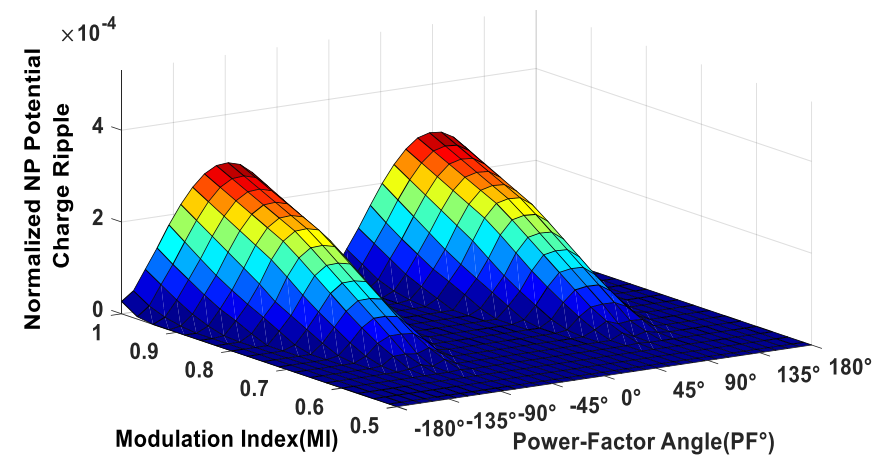

Fig. 6. Normalized magnitude of NP potential charge ripple.

vector. Here, a four-state NTV scheme [8] is chosen to explain the synthesis process in the space vector diagram (SVD).

As shown in Fig.4, when the reference voltage vector falls into triangle 3 of Sector-1, it can be composed by the nearestthree space vectors labeled with numbers 1-3. The switching sequence is $[\mathrm{POO}] \rightarrow[\mathrm{PON}] \rightarrow[\mathrm{OON}] \rightarrow[\mathrm{ONN}] \rightarrow[\mathrm{OON}] \rightarrow$ $[\mathrm{PON}] \rightarrow[\mathrm{POO}]$. Similarly, the nearest-three space vectors labeled with number 1, 3 and 4 can synthesize the reference voltage vector located in triangle 5, the corresponding switching sequence is arranged as $[\mathrm{POO}] \rightarrow[\mathrm{PON}] \rightarrow[\mathrm{PNN}] \rightarrow$ $[\mathrm{ONN}] \rightarrow[\mathrm{PNN}] \rightarrow[\mathrm{PON}] \rightarrow[\mathrm{POO}]$.

In accordance with the volts-second principle, the following expressions can be given as:

$$
\left\{\begin{array}{l}
V_{\text {ref }} T_{s}=V_{1} T_{1}+V_{2} T_{2}+V_{3} T_{3} \\
T_{s}=T_{1}+T_{2}+T_{3}
\end{array}\right.
$$

where $T_{s}$ is the switching period. $T_{1}, T_{2}$ and $T_{3}$ denote the dwell time of $V_{l}, V_{2}$ and $V_{3}$, respectively.

For example, when $V_{\text {ref }}$ is located in triangle 6 of Sector-1, the duty cycle can be calculated as: 


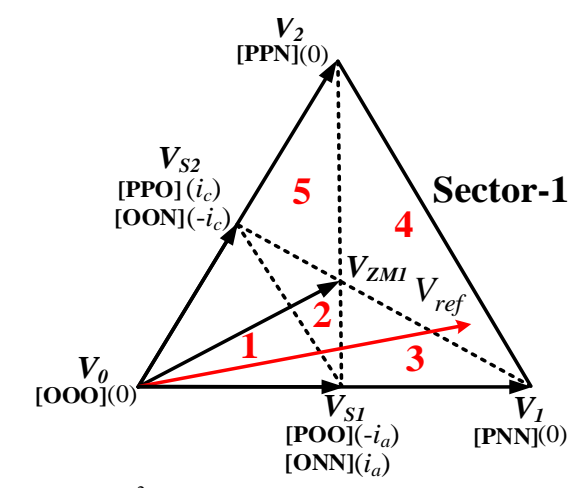

Fig. 7. SVD of the $\mathrm{NTV}^{2}$ scheme in Sector-1.

$$
\left\{\begin{array}{l}
d_{1}=2 m \sin (\theta)-1 \\
d_{2}=2 m \sin (\pi / 3-\theta) \\
d_{3}=2[1-m \sin (\pi / 3+\theta)]
\end{array}\right.
$$

where $m$ denotes the modulation index and $\theta$ indicates the azimuth angle of $V_{\text {ref }}$.

In order to reveal the participation of small and medium vectors at different modulation indices, the duty cycles of these vectors over a line-frequency period are plotted in Fig.5. It can be clearly seen that medium vector becomes predominant along with increasing modulation index.

Hence, for the reference space vector in Fig. 4, the average NP current generated by this method can be expressed as:

$$
\overline{I_{N P}}=\left(d_{o n n}-d_{p o o}\right) \cdot i_{a}+d_{p o n} \cdot i_{b}+\left(d_{p p o}-d_{o o n}\right) \cdot i_{c}
$$

Using a similar method for all the sectors in a fundamental period, the NP voltage variation can be thus derived by:

$$
\Delta V_{N P}=\frac{1}{C} \int_{t=0}^{t=T_{s}} \overline{I_{N P}} \cdot d t
$$

where $C$ represents the DC-link capacitance.

With (3) and (4), the undesirable NP potential charge ripple is shown in Fig.6. As shown, the NP voltage unbalance by the NTV scheme is much aggravated when the modulation index approaches unity and power factor angle towards $90^{\circ}$. Noted that the DC component of this oscillation drifts capacitor voltage away, whereas its $\mathrm{AC}$ components have a negative impact on the lifetime of DC-link capacitors. As the studied 3LNPC converter-fed PMSM drives exhibit high modulation index and low power factor, NP voltage imbalance issue in our case should be paid more attention.

\section{B. Conventional NTV ${ }^{2}$}

The conventional $\mathrm{NTV}^{2}$ scheme presented by Sergio Busquets-Monge et al. [11] is comprised of a series of virtual space vectors. Considering that all employed virtual space vectors produce average zero NP current. This technique is capable of solving the NP voltage drift problem for all loads. The specific definition is expressed as follows:

$$
\left\{\begin{array}{l}
V_{S 1}=\left(V_{P O O}\left[-i_{a}\right]+V_{O N N}\left[i_{a}\right]\right) / 2 \\
V_{S 2}=\left(V_{P P O}\left[i_{c}\right]+V_{O O N}\left[-i_{c}\right]\right) / 2 \\
V_{M 1}=\left(V_{O N N}\left[i_{a}\right]+V_{P O N}\left[i_{b}\right]+V_{P P O}\left[i_{c}\right]\right) / 3 \\
V_{0}=V_{O O O}[0] \\
V_{L 1}=V_{P N N}[0] \\
V_{L 2}=V_{P P N}[0]
\end{array}\right.
$$

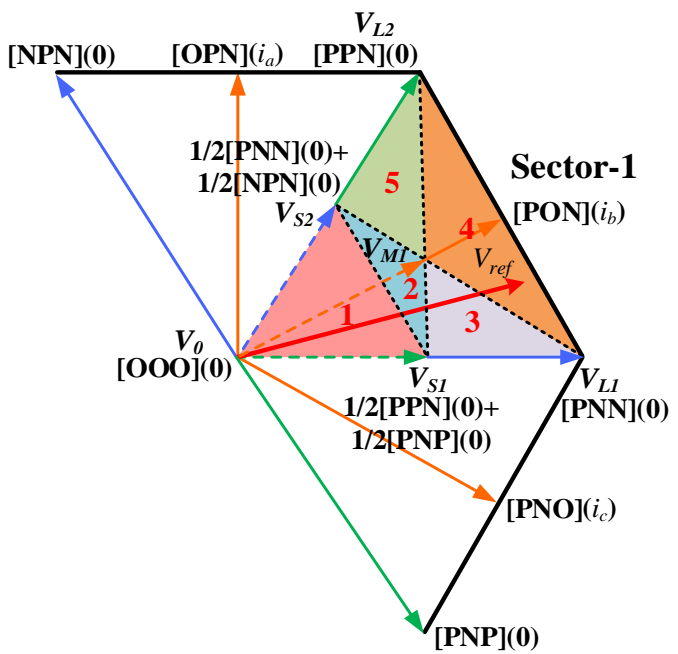

Fig.8. SVD of the proposed modulation strategy in Sector-1.

TABLE II

CMV ANALYSIS OF SWITCHING STATES AND SPACE VeCTORS

\begin{tabular}{ccc}
\hline \hline Space Vectors & Switching States & $\left|V_{c o m}\right|$ \\
\hline \multirow{2}{*}{ Zero Vector } & {$[\mathrm{OOO}]$} & 0 \\
& {$[\mathrm{PPP}][\mathrm{NNN}]$} & $V_{d c} / 2$ \\
\hline \multirow{2}{*}{ Small Vector } & {$[\mathrm{POO}][\mathrm{OON}][\mathrm{OPO}][\mathrm{NOO}][\mathrm{OOP}][\mathrm{ONO}]$} & $V_{d c} / 6$ \\
& {$[\mathrm{ONN}][\mathrm{PPO}][\mathrm{NON}][\mathrm{OPP}][\mathrm{NNO}][\mathrm{POP}]$} & $V_{d c} / 3$ \\
\hline Medium Vector & {$[\mathrm{PON}][\mathrm{OPN}][\mathrm{NPO}][\mathrm{NOP}][\mathrm{ONP}][\mathrm{PNO}]$} & 0 \\
\hline Large Vector & {$[\mathrm{PNN}][\mathrm{PPN}][\mathrm{NPN}][\mathrm{NPP}][\mathrm{NNP}][\mathrm{PNP}]$} & $V_{d c} / 6$ \\
\hline \hline
\end{tabular}

As can be seen from (5), the shifted capacitor voltage can be naturally mitigated by using proportionally synthesized small vectors $V_{s 1}, V_{s 2}$ and medium vector $V_{M 1}$. Assuming that $V_{r e f}$ is located in triangle 4 of the first sector, as shown in Fig.7, the switching sequence will be $[\mathrm{PPO}] \rightarrow[\mathrm{PPN}] \rightarrow[\mathrm{PON}] \rightarrow[\mathrm{PNN}] \rightarrow$ $[\mathrm{ONN}] \rightarrow[\mathrm{PNN}] \rightarrow[\mathrm{PON}] \rightarrow[\mathrm{PPN}] \rightarrow[\mathrm{PPO}]$. It can be seen that the $\mathrm{NTV}^{2}$ scheme produces more switching actions during a switching cycle. More specifically, the NTV scheme has 6 switching actions, whereas this approach features 8 switching actions. As a result, the $\mathrm{NTV}^{2}$ method achieves NP voltage balance at the expense of more switching losses. Furthermore, the original modulation scheme fails to consider the CMV. This is critical for high-speed PMSM fed high-switching-frequency converter in the ESG system since that value will bring in EMI issues for avionics of aircraft.

\section{PROposed Modulation StRATEGY}

In this section, a modified $\mathrm{NTV}^{2}$ strategy that enables to attain the NP voltage balance and CMV reduction is presented in the following subsections.

\section{A. CMV Analysis}

The CMV is the voltage between the midpoint of load and the NP of DC-link, which is defined by:

$$
V_{c o m}=\frac{1}{3}\left(V_{a o}+V_{b o}+V_{c o}\right)
$$

where $V_{a o}, V_{b o}$ and $V_{c o}$ are the phase voltage with respect to the NP potential.

Through the calculation, the CMV of all switching states is listed in Table II. As shown, CMVs contain a series of DC voltage that is the source of bearing current in motor drives. Moreover, it causes winding deterioration and even failure. 


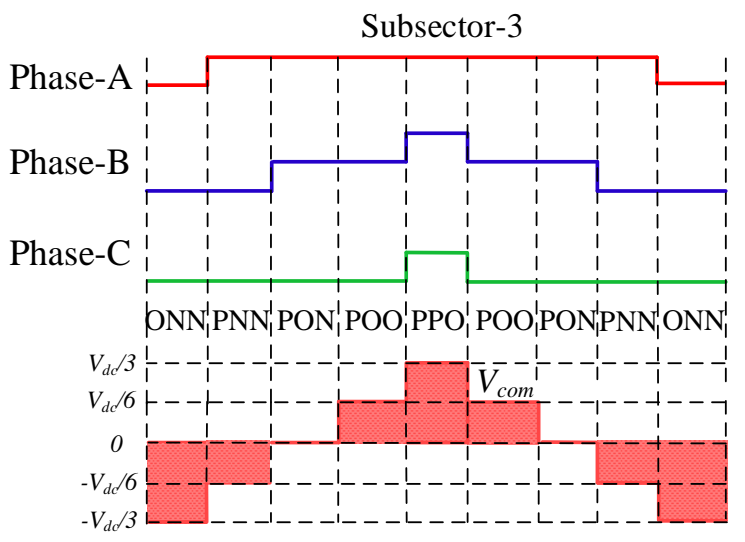

(a)

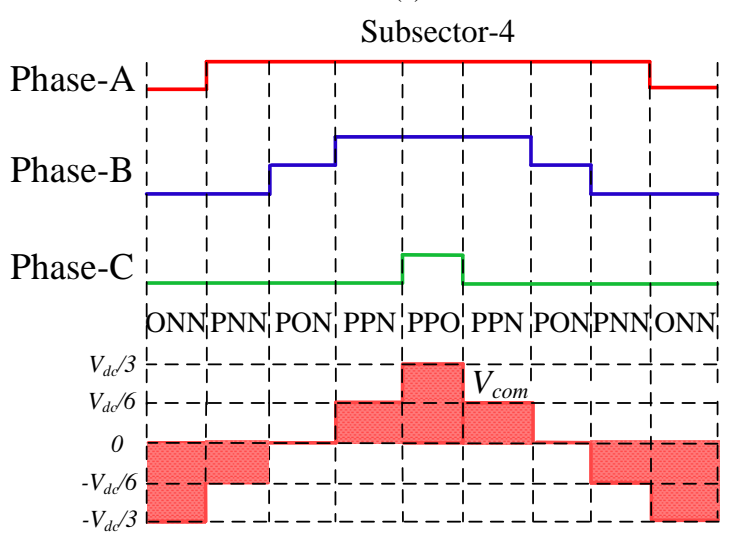

(b)

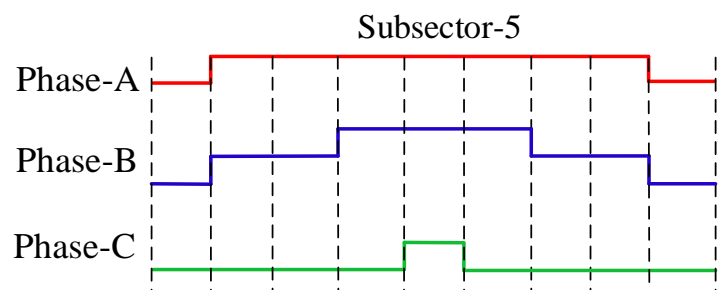

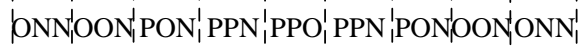

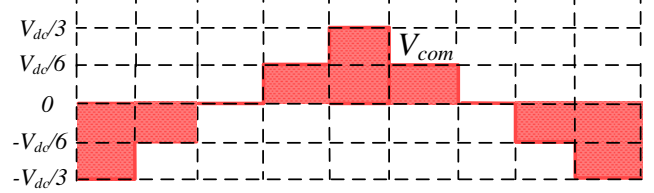

(c)

Fig.9. Switching sequence and CMV of the $\mathrm{NTV}^{2}$ strategy in the generation mode. (a) Subsector-3. (b) Subsector-4. (c) Subsector-5.

\section{B. Proposed Virtual Space Vector}

To realize balanced capacitor voltage and reduced CMV for the target ESG system, the principle for selecting a switching state needs to satisfy the following requirements so that the modified virtual space vectors are able to achieve advantages over the conventional $\mathrm{NTV}^{2}$ scheme.

1) The sum of NP currents associated with synthesized virtual space vectors should be average zero.

2) The CMVs produced by basic space vectors should be as low as possible.

Therefore, a straightforward algorithm is that two pairs of large vectors are employed to compose two virtual small vectors and adjacent three medium vectors synthesize one virtual medium vector, whereas the selection of zero and large vector still follow the same rule of the conventional $\mathrm{NTV}^{2}$

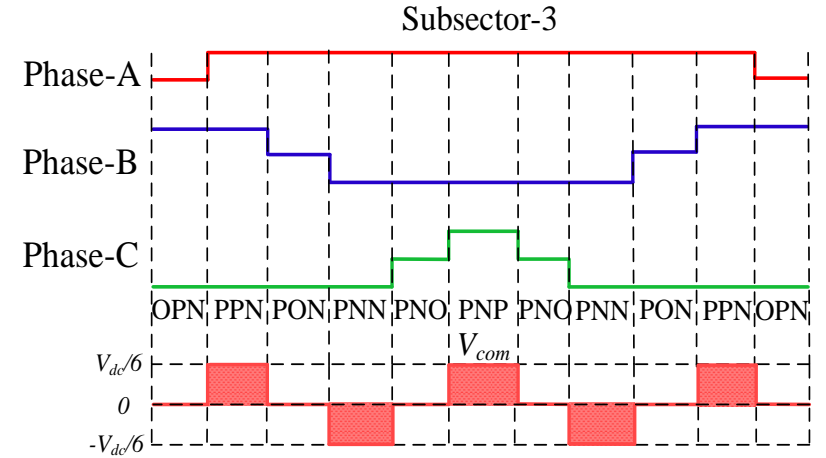

(a)

Subsector-4

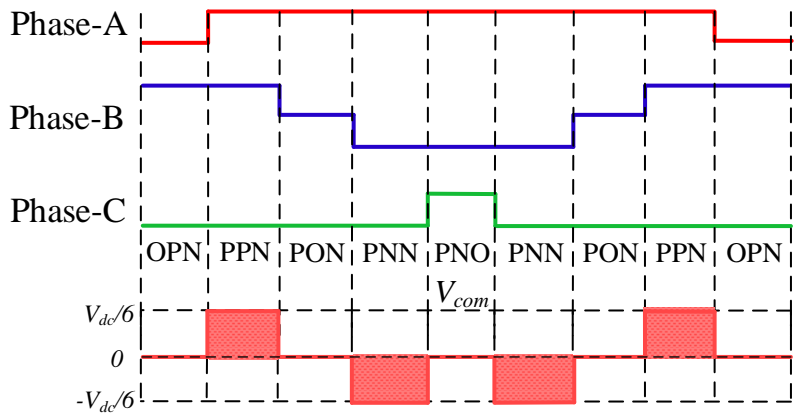

(b)

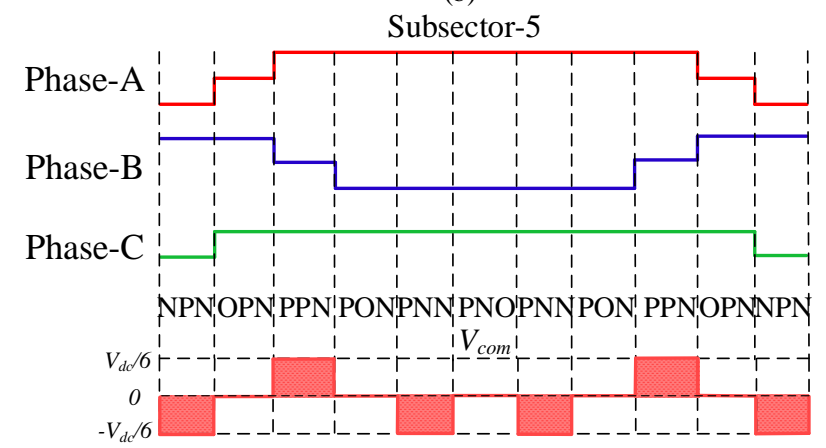

(c)

Fig.10. Switching sequence and CMV of the proposed modulation strategy in the generation mode. (a) Subsector-3. (b) Subsector-4. (c) Subsector-5.

scheme. This forms the basis of the enhanced virtual space vector modulation scheme in this paper. In addition, it should be noted that, although omitting the composed virtual small vectors can simplify reference voltage vector approximation, non-NTV is unfavorable to output harmonic performance [34], particularly for the wide-speed range operation of ESG system.

As illustrated in Fig.8, the blue, orange, and green dashed arrow lines indicates these modified virtual space vectors that are defined by:

$$
\left\{\begin{array}{l}
V_{S 1}=\left(V_{P P N}[0]+V_{P N P}[0]\right) / 2 \\
V_{S 2}=\left(V_{P N N}[0]+V_{N P N}[0]\right) / 2 \\
V_{M 1}=\left(V_{O P N}\left[i_{a}\right]+V_{P O N}\left[i_{b}\right]+V_{P N O}\left[i_{c}\right]\right) / 3 \\
V_{0}=V_{O O O}[0] \\
V_{L 1}=V_{P N N}[0] \\
V_{L 2}=V_{P P N}[0]
\end{array}\right.
$$

Taking the same example in Section III. B, i.e., $V_{\text {ref }}$ falls into triangle 4 of Sector-1, the switching sequence within a PWM cycle can be assigned as $[\mathrm{OPN}] \rightarrow[\mathrm{PPN}] \rightarrow[\mathrm{PON}] \rightarrow$ $[\mathrm{PNN}] \rightarrow[\mathrm{PNO}] \rightarrow[\mathrm{PNN}] \rightarrow[\mathrm{PON}] \rightarrow[\mathrm{PPN}] \rightarrow[\mathrm{OPN}] . \mathrm{As}$ 


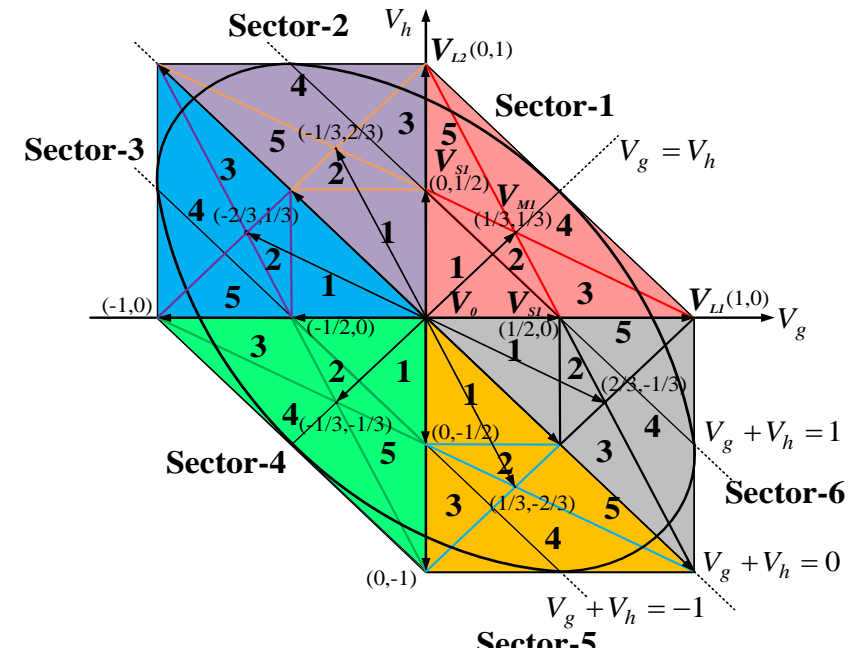

Fig. 11. Modified SVD of the proposed modulation strategy. TABLE III

THE SUBSECTOR IDENTIFICATION OF SECTOR-1

\begin{tabular}{cc}
\hline \hline Subsector & Identification Criteria \\
\hline 1 & $0<V_{g}+V_{h} \leq 0.5$ \\
2 & $V_{g}+V_{h}>0.5 \& \& V_{h}+2 V_{g} \leq 1 \& \& 0.5 V_{g}+V_{h} \leq 0.5$ \\
3 & $V_{h}+2 V_{g}>1 \& \& 0.5 V_{g}+V_{h}<0.5 \& \& V_{h} \geq 0$ \\
4 & $V_{g}+V_{h}<1 \& \& V_{h}+2 V_{g} \geq 1 \& \& 0.5 V_{g}+V_{h} \geq 0.5$ \\
5 & $V_{g} \geq 0 \& \& 0.5 V_{g}+V_{h}>0.5 \& \& V_{h}+2 V_{g} \leq 1$ \\
\hline \hline
\end{tabular}

TABLE IV

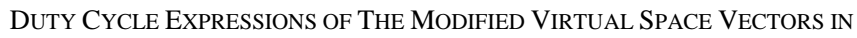
EACH SUBSECTOR OF SECTOR-1

\begin{tabular}{cccc}
\hline \hline Subsector & $d_{1}$ & $d_{2}$ & $d_{3}$ \\
\hline 1 & $2 g$ & $2 h$ & $1-2(g+h)$ \\
2 & $2(1-g-2 h)$ & $2(1-h-2 g)$ & $3[2(g+h)-1]$ \\
3 & $2(1-2 h-g)$ & $2 g+h-1$ & $3 h$ \\
4 & $3(1-g-h)$ & $2 g+h-1$ & $2 h+g-1$ \\
5 & $2(1-2 g-h)$ & $2 h+g-1$ & $3 g$ \\
\hline \hline
\end{tabular}

TABLE V

Duty CyCle of Basic Space Vectors In SubSector 3-5 OF SeCtor-1

\begin{tabular}{ccc}
\hline \hline Subsector & Space Vectors & Duty Cycle \\
\hline & {$[\mathrm{PNP}]$} & $1-g-2 h$ \\
& {$[\mathrm{PNO}]$} & $h$ \\
3 & {$[\mathrm{PNN}]$} & $2 g+h-1$ \\
& {$[\mathrm{PON}]$} & $h$ \\
& {$[\mathrm{PPN}]$} & $1-g-2 h$ \\
& {$[\mathrm{OPN}]$} & $h$ \\
\hline & {$[\mathrm{OPN}]$} & $1-g-h$ \\
& {$[\mathrm{PNN}]$} & $2 h+g-1$ \\
4 & {$[\mathrm{PON}]$} & $1-g-h$ \\
& {$[\mathrm{PNN}]$} & $2 g+h-1$ \\
& {$[\mathrm{PNO}]$} & $1-g-h$ \\
\hline & {$[\mathrm{PNO}]$} & $g$ \\
& {$[\mathrm{PPN}]$} & $1-h-2 g$ \\
& {$[\mathrm{PON}]$} & $g$ \\
5 & {$[\mathrm{PPN}]$} & $2 h+g-1$ \\
& {$[\mathrm{OPN}]$} & $g$ \\
& {$[\mathrm{NPN}]$} & $1-h-2 g$ \\
\hline \hline
\end{tabular}

mentioned before, power generation mode is the steady-state of the aircraft ESG system. Consequently, $V_{\text {ref }}$ is primarily synthesized by modified virtual space vectors in subsector 3-5 of each sector. The CMVs generated by the original $\mathrm{NTV}^{2}$ and the proposed modulation strategy are shown in Fig.9 and Fig.10, respectively. As can be seen, compared with the $\mathrm{NTV}^{2}$ scheme, CMVs are reduced to half by the proposed technique, i.e., within one-sixth of DC-link voltage. Furthermore, it is worth noting that the presented approach brings a chance to address

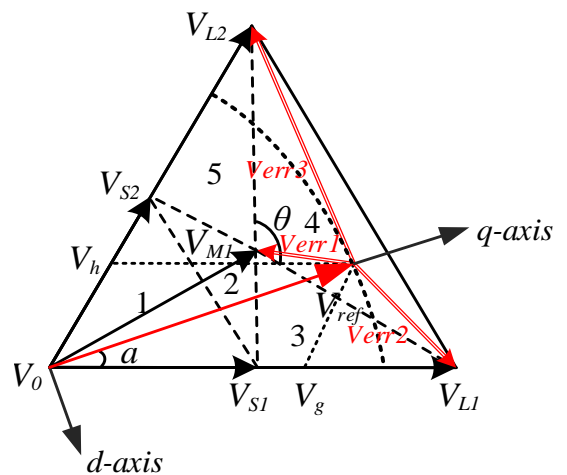

Fig. 12. The voltage error vectors associated with virtual space vectors.

the NP voltage drift issue irrespective of modulation index and load angle.

\section{The Fast Calculation Approach}

Apart from modulation strategy performed in the PWM modulator, other crucial control algorithms need to be executed within a PWM interrupt service routine (ISR). As the sampling time of the applied controller is only $62.5 \mu \mathrm{s}$, a fast digital implementation is necessarily considered to overcome the computational burden. In comparison with the traditional orthogonal $\alpha-\beta$ frame, the hexagonal SVD is modified via:

$$
\left[\begin{array}{l}
V_{g} \\
V_{h}
\end{array}\right]=\left[\begin{array}{cc}
1 & -\frac{1}{\sqrt{3}} \\
0 & \frac{2}{\sqrt{3}}
\end{array}\right]\left[\begin{array}{l}
V_{\alpha} \\
V_{\beta}
\end{array}\right]
$$

All virtual space vectors are shown in Fig.11. Noteworthy, the scale of all space vectors is normalized with respect to the magnitude of the large vector. For example, the coordinator of $V_{L I}$ is represented as $(1,0)$. Thus, each boundary line can be easily attained. With the linear programming, the subsector identification criteria in Sector-1 are subsequently detailed in Table III. The duty cycle of the modified virtual space vectors is found in Table IV. The duty cycle of the used basic space vector is listed in Table V. It is clear that, unlike (2), the expressions of duty cycle are simplified by algebraic functions.

In addition, with (9), reference voltage vector located in other sectors can be converted into the first sector so that iterative judgment processes can be effectively avoided.

$$
\left[\begin{array}{c}
V_{g 1} \\
V_{h 1}
\end{array}\right]=\left[\begin{array}{cc}
1 & 1 \\
-1 & 0
\end{array}\right]^{(N-1)}\left[\begin{array}{c}
V_{g N} \\
V_{h N}
\end{array}\right]
$$

where $N$ represents the sector number.

Therefore, the subsector identification and duty cycle determination presented in Table III and Table IV are sufficient to generate dedicated pulse train over a fundamental period.

\section{Torque Ripple Analysis}

In this subsection, the torque ripple produced by the proposed technique is analyzed. The original $\mathrm{NTV}^{2}$ scheme is selected as the benchmark method to compare that performance.

The electromagnetic torque of the applied surface-mounted PMSM is expressed as:

$$
T_{e m}=\frac{3}{2} n_{p} \psi_{f} i_{q}
$$

where $n_{p}$ is the pole pairs and $\psi_{f}$ refers to the magnet flux. 

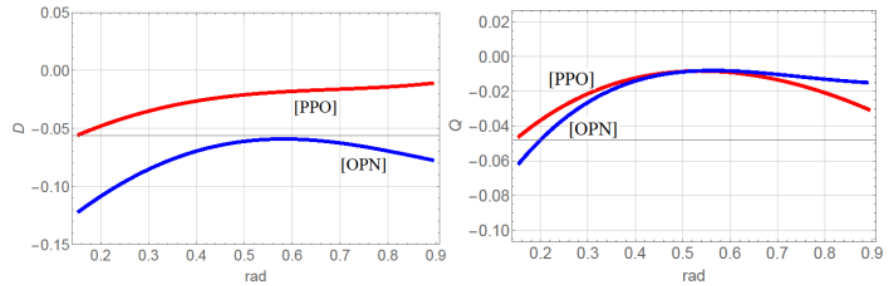

(a)
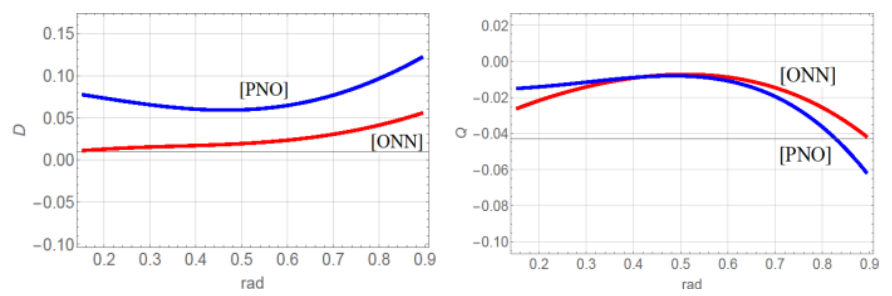

(b)
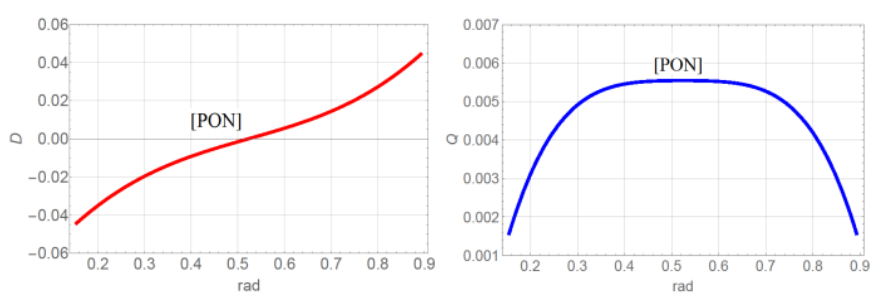

(c)

Fig.13. $D-Q$ components of stator flux ripple vector caused by different switching states of the benchmark method and the proposed modulation scheme in subsector-4. (a) [PPO] and [OPN]. (b) [ONN] and [PNO]. (c) [PON].

As $n_{p}$ and $\psi_{f}$ are constant values for the PMSM, the torque ripple is proportional to $q$-axis current ripple. As shown in Fig.12, the direction of $V_{r e f}$ and its clockwise perpendicular line is defined as $q$ - and $d$-axis, respectively. $\alpha$ is the angle between $V_{r e f}$ and $V_{L l}$. In addition to zero vectors, other virtual vectors can be expressed based on this predefined frame. It is worth noting that all space vectors are normalized with the magnitude of $V_{L l}$. By doing so, the following equations can be obtained:

$$
\begin{gathered}
V_{S 1}=\frac{1}{2} \sin \alpha+\frac{1}{2} \cos \alpha j \\
V_{L 1}=\sin \alpha+\cos \alpha j \\
V_{S 2}=-\frac{1}{2} \sin \left(60^{0}-\alpha\right)+\frac{1}{2} \cos \left(60^{0}-\alpha\right) j \\
V_{L 2}=-\sin \left(60^{0}-\alpha\right)+\cos \left(60^{0}-\alpha\right) j \\
\left\{\begin{array}{l}
\sqrt{3} \\
V_{M 1}=\frac{\sin \left(30^{0}-\alpha\right)}{\sqrt{3}}+\frac{\cos \left(30^{0}-\alpha\right) j}{\sqrt{3}} \alpha \in\left(0,30^{0}\right] \\
V_{M 1}=\frac{\sin \left(\alpha-30^{0}\right)}{\sqrt{3}}+\frac{\cos \left(\alpha-30^{0}\right) j}{\sqrt{3}} \quad \alpha \in\left(30^{\circ}, 60^{\circ}\right]
\end{array}\right.
\end{gathered}
$$

Without loss of generality, subsector-4 shown in Fig.12 is considered as a representative example to analyze the torque ripple in the following. Thus, $\alpha$ is constrained by:

$\arcsin \left(\frac{1}{m \sqrt{3}}\right)-30^{\circ}<\theta<90^{\circ}-\arcsin \left(\frac{1}{m \sqrt{3}}\right) m \in[0.67,1]$

As the time integral of error space vectors between $V_{\text {ref }}$ and three virtual space vectors shown in Fig. 11 refer to stator flux ripple vectors, which is a measurement of ripple in the line current [35]. Then, (12), (14) and (15) can be further derived as:

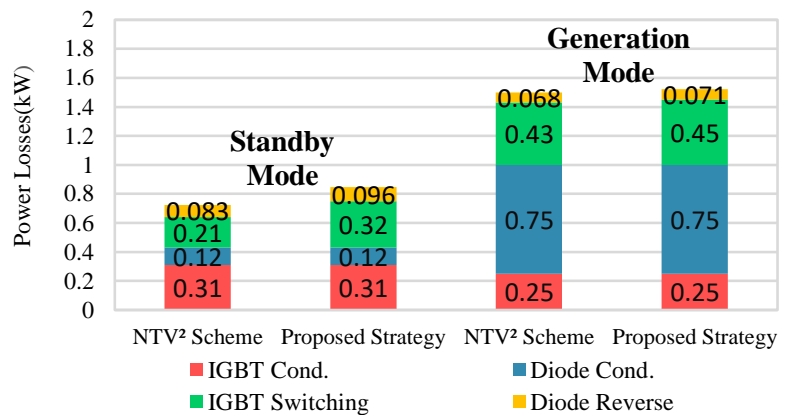

Fig.14. Comparison of power losses distribution of the $\mathrm{NTV}^{2}$ scheme and the proposed modulation strategy under two operating conditions.

$$
\begin{aligned}
& \psi_{e r r 1}=-\frac{t_{1} \sin \left(30^{0}-\alpha\right)}{\sqrt{3}}+t_{1}\left[\frac{1}{\sqrt{3}} \cos \left(30^{0}-\alpha\right)-V_{r e f}\right] j \\
& \alpha \in\left(\arcsin \left[\frac{1}{m \sqrt{3}}\right]-30^{0}, 30^{\circ}\right] \\
& \left\{\begin{array}{l}
\psi_{\text {err } 1}=\frac{t_{1} \sin \left(\alpha-30^{0}\right)}{\sqrt{3}}+t_{1}\left[\frac{1}{\sqrt{3}} \cos \left(\alpha-30^{0}\right)-V_{r e f}\right] j \\
\alpha \in\left(30^{0}, 90^{0}-\arcsin \left[\frac{1}{m \sqrt{3}}\right]\right)
\end{array}\right. \\
& \left\{\begin{array}{l}
\psi_{\text {err } 2}=t_{2} \sin \alpha+t_{2}\left(\cos \alpha-V_{r e f}\right) j \\
\psi_{\text {err } 3}=-t_{3} \sin \left(60^{\circ}-\alpha\right)+t_{3}\left[\cos \left(60^{0}-\alpha\right)-V_{r e f}\right] j
\end{array}\right.
\end{aligned}
$$

where $t_{1 \sim 3}$ represents the dwell time of $V_{M 1}, V_{S 1}$ and $V_{S 2}$.

In terms of the proposed approach, flux ripple vectors produced by large vectors are the same as the conventional $\mathrm{NTV}^{2}$ scheme. However, the error vectors generated by medium virtual space vectors are distinctively different. The flux ripple vector produced by [PPO], [ONN] and [PON] are yielded as:

$$
\begin{aligned}
& \left\{\begin{array}{l}
\psi_{\text {ppo }}^{\text {err }}=-\frac{1}{2} t_{1} \sin \left(60^{0}-\alpha\right)+t_{1}\left[\frac{1}{2} \cos \left(60^{\circ}-\alpha\right)-V_{r e f}\right] j \\
\psi_{\text {onn }}^{\text {err }}=\frac{1}{2} t_{1} \sin \alpha+t_{1}\left(\frac{1}{2} \cos \alpha-V_{r e f}\right) j
\end{array}\right. \\
& \left\{\begin{array}{l}
\psi_{\text {pon }}^{\text {err }}=-\frac{t_{1} \sqrt{3} \sin \left(30^{0}-\alpha\right)}{2}+t_{1}\left[\frac{\sqrt{3}}{2} \cos \left(30^{0}-\alpha\right)-V_{r e f}\right] j \\
\alpha \in\left(\arcsin \left[\frac{1}{m \sqrt{3}}\right]-30^{0}, 30^{0}\right] \\
\psi_{\text {pon }}^{\text {err }}=-\frac{t_{1} \sqrt{3} \sin \left(\alpha-30^{0}\right)}{2}+\left[\frac{\sqrt{3}}{2} \cos \left(\alpha-30^{0}\right)-V_{r e f}\right] j \\
\alpha \in\left(30^{0}, 90^{0}-\arcsin \left[\frac{1}{m \sqrt{3}}\right]\right)
\end{array}\right.
\end{aligned}
$$

Compared (19) and (20) with (17), the flexible rotation coefficient $\mathfrak{R}$ is given by (21), which can be used to evaluate the current ripple produced by all virtual space vectors.

$$
\mathfrak{R}=A \times e^{ \pm j \zeta}=\left|\frac{V_{\text {basic }}}{V_{\text {virtual }}}\right| \times e^{ \pm j \zeta}
$$

where $A$ and $\zeta$ represent length ratio and the angle between basic and virtual space vector, respectively. Noted that the counterclockwise direction determines positive sign above.

Further, the flux error can be defined by:

$$
\psi_{\text {err }}=D+Q \cdot j
$$

As a result, current ripple caused by the modulation scheme 
TABLE VI

ESG SYSTEM PARAMETERS

\begin{tabular}{cc}
\hline \multicolumn{2}{c}{ ESG SYSTEM PARAMETERS } \\
\hline Parameters & Value \\
\hline$L_{d}=L_{q}$ & $99 \mu \mathrm{H}$ \\
Pole pair & 3 \\
Switching frequency & $16 \mathrm{kHz}$ \\
PM flux & $0.03644 \mathrm{~V}_{\mathrm{s}} / \mathrm{rad}$ \\
Base speed & $8000 \mathrm{rpm}$ \\
Fundamental frequency in generation mode & $1 \sim 1.6 \mathrm{kHz}$ \\
Capacitor Value $\left(C_{l}=C_{2}\right)$ & $600 \mu \mathrm{F}$ \\
DC-link Voltage & $270 \mathrm{~V}$ \\
\hline \hline
\end{tabular}

can be evaluated by $D$ and $Q$. The amplitude of $D-Q$ component of stator flux ripple vector is shown Fig.13. As can be seen, the value of the proposed solution is a bit larger, which leads to a higher $q$-axis current ripple. Similar derivations and analyses can be carried out in other subsectors. Overall, the torque ripple produced by the modified strategy is inevitably increased. It is thus a trade-off to balance two capacitor voltages and reduce CMV for high-speed motor drives, simultaneously.

\section{E. Power Losses Analysis}

The power losses of the converter consist of conduction and switching losses. The former one is mainly related to the current flowing through switches, whereas the latter depends on the number of commutations during a switching period.

It is known that the presented modulation technique force some phases to clamp to the positive/negative DC-rail and the NP. In particular, when reference voltage vector is synthesized in subsector-2, twelve commutations are conducted over a switching cycle, which results in extra $50 \%$ switching losses compared with the conventional $\mathrm{NTV}^{2}$ scheme. Nonetheless, in the steady-state of the studied ESG system, space vector is primarily composed in subsector-4, which features eight turnon/off actions. Thus, power losses of the proposed scheme are similar to that of the benchmark method in generation mode.

The power losses are calculated by the thermal model of applied Infineon F3L400R07ME4_B22/B23 insulated-gate bipolar transistor (IGBT) module. Here, the converter loss by the conventional $\mathrm{NTV}^{2}$ scheme and the presented modulation strategy, as shown in Fig.13, is carried out when the system operates in standby mode and power generating condition of 25 $\mathrm{kW}$ at $20 \mathrm{krpm}$. The switching frequency is set at $16 \mathrm{kHz}$.

\section{SimUlation AND EXPERIMENTAL RESUlTS}

\section{A. Simulation Results}

The simulated model of the ESG system shown in Fig.3 is built in Simulink/PLECS environment and the parameters are given in Table VI. In simulation setup, the system initially operates in the startup process, and then flux-weakening control starts at $0.45 \mathrm{~s}$. After that, the engine ignites. Regenerative operation is activated by connecting a $3 \mathrm{~kW}$ resistive load to the DC-link at $1 \mathrm{~s}$ with a machine speed of $20 \mathrm{krpm}$, and then the droop control takes over DC-link voltage regulation at the same time. At 1.1s, PWM modulator switches from the conventional $\mathrm{NTV}^{2}$ scheme to the proposed modulation strategy. At that moment, average modulation index and power factor are 0.95 and 0.15, respectively. In Fig.15, the PMSM speed diagram describes the ESG system status during the entire speed range operation. As observed from Fig.16 and Fig.17, not only the

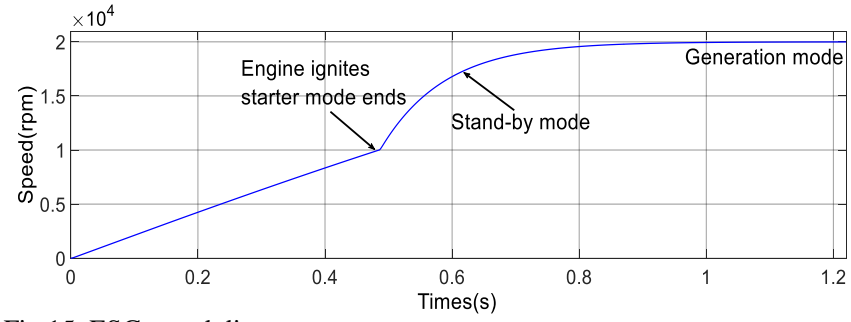

Fig.15. ESG speed diagram.

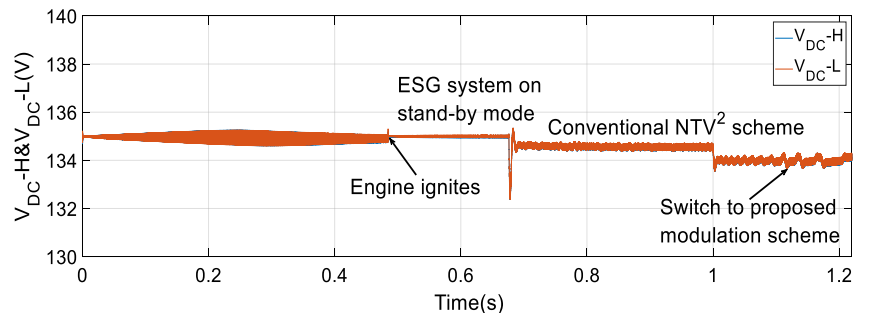

Fig.16. DC-link capacitor voltage of the ESG system under conventional NTV 2 and the proposed modulation schemes.

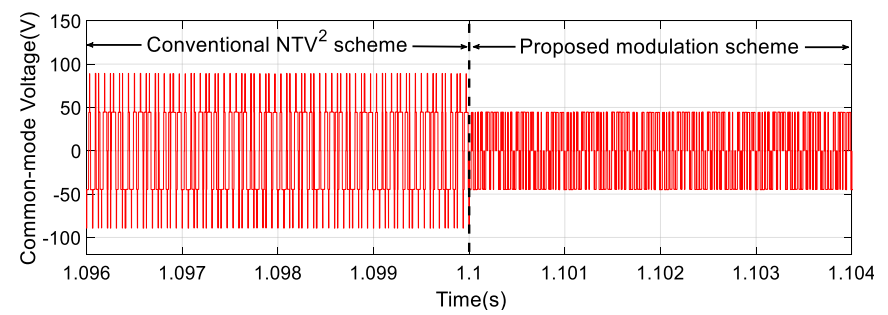

Fig. 17. CMV comparison of the ESG system under conventional $\mathrm{NTV}^{2}$ and the proposed modulation schemes.

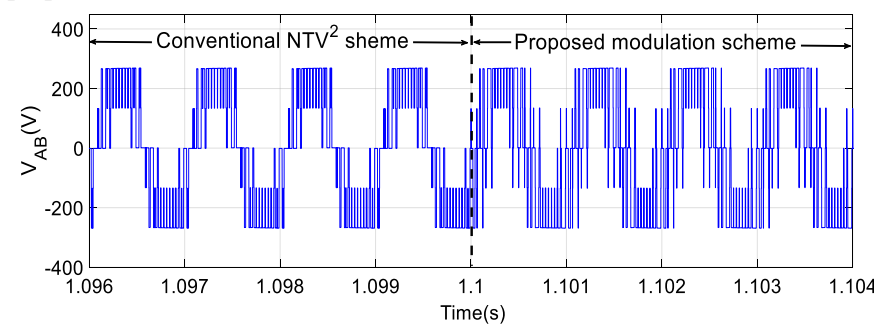

Fig.18. Line-to-line voltage during the modulation algorithm transition period.

proposed approach can balance NP potential, but also the CMV is effectively reduced to half of the prior value $\left( \pm 1 / 6 V_{d c}\right)$.

Fig.18 shows the line-to-line voltage $\left(V_{A B}\right)$ during the algorithm transition period. As shown, the three-level switching actions of the proposed modulation scheme are more than that by the $\mathrm{NTV}^{2}$ scheme. Fig.19 gives harmonic spectra comparison of the phase current $\left(i_{a}\right)$ between $\mathrm{NTV}^{2}$ scheme and the proposed method in generation mode. It can be seen that, with the proposed strategy applied, the harmonic components are lower than that of the counterpart near fundamental frequency $(1 \mathrm{kHz})$, whereas the value becomes large and around the switching frequency $(16 \mathrm{kHz})$. Moreover, the modified scheme introduces a total harmonic distortion (THD) of $5.43 \%$, which is higher than the $\mathrm{NTV}^{2}$ scheme of $4.78 \%$.

\section{B. Experimental Implementations and Results}

The proposed modulation strategy is verified by a $45 \mathrm{~kW}, 32$ $\mathrm{krpm}$ aircraft ESG prototype system. The hardware of the test rig is shown in Fig.20. A $150 \mathrm{~kW}$ prime mover and high-speed machine are placed in an isolated room for safety consideration. The prime mover mechanically emulates aircraft engine shaft that is coupled to the high-speed PMSM. DSK6713 and Actel 

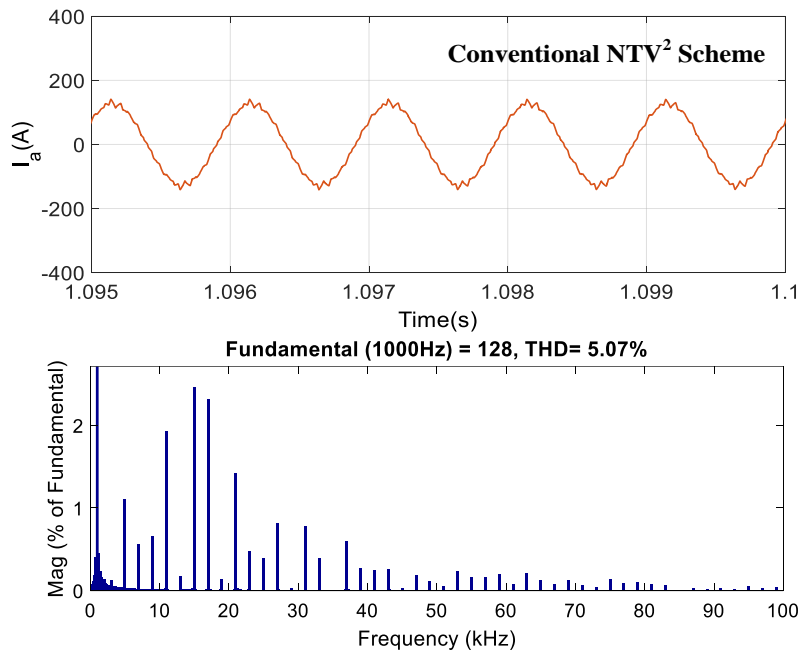

(a)
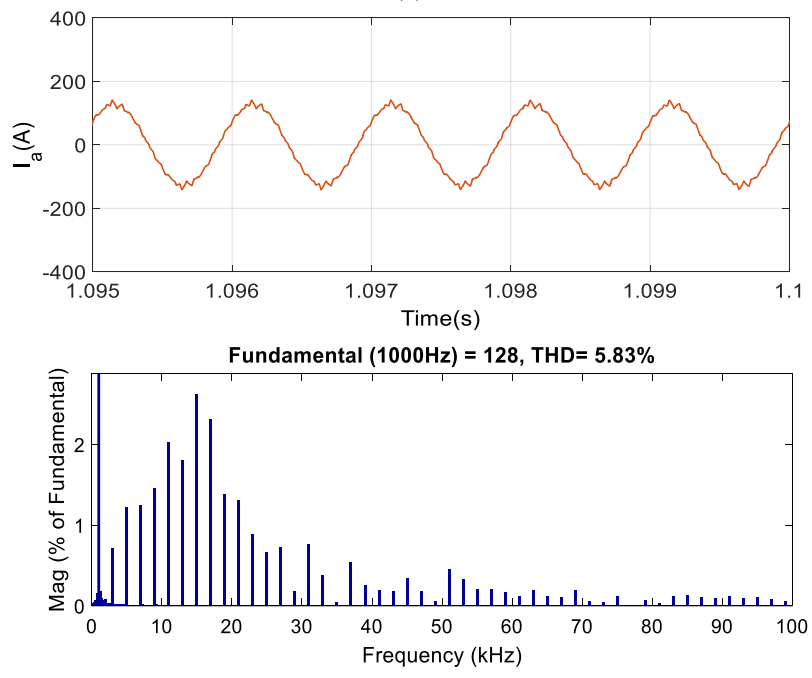

(b)

Fig.19. Phase current $\left(i_{a}\right)$ and its harmonic spectra in the generation mode. (a) The conventional $\mathrm{NTV}^{2}$ scheme. (b) The proposed modulation scheme.

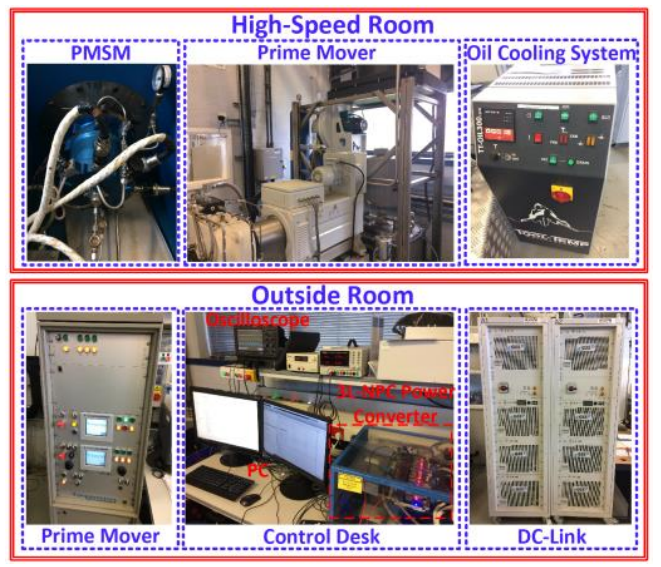

Fig.20. Experimental test rig.

FPGA-ProAsic3 A3P400 are chosen as the control platform. AC-side parameters are monitored by PPA5530 power analyzer. Considering that phase current direction changes rapidly at light-load condition, it aggravates the NP voltage drift problem. Therefore, the experimentations mainly focus that the ESG system operates at a light-load condition during generation mode, whereas the startup process is not presented here as it

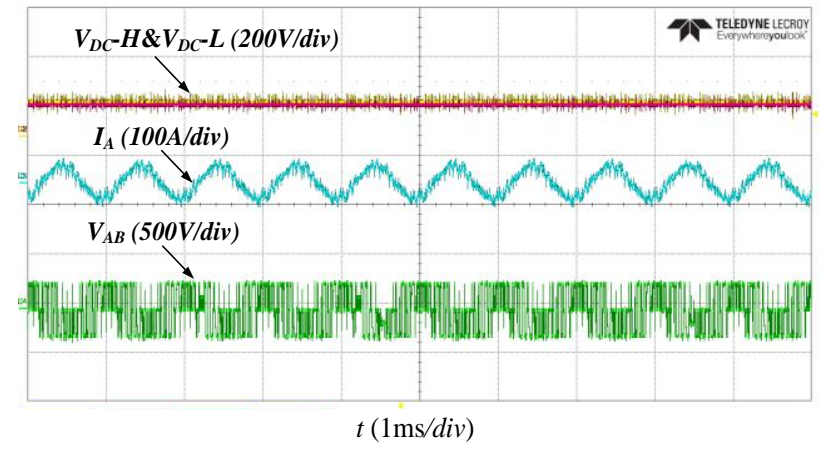

(a)

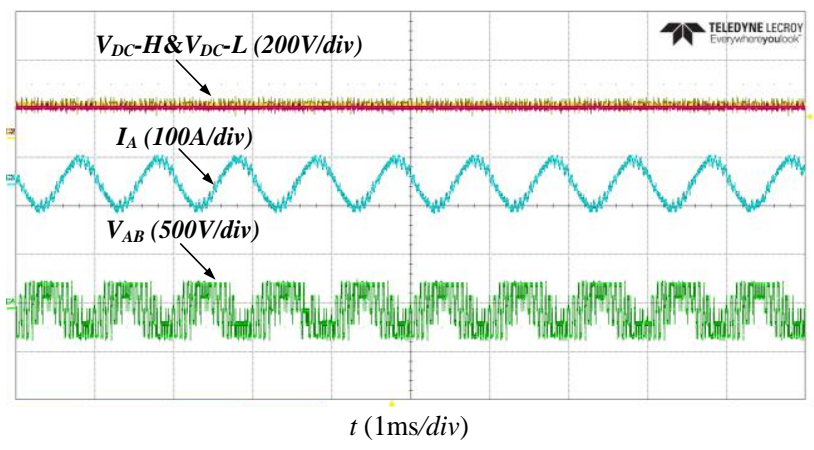

(b)

Fig.21. Experimental results of the proposed modulation strategy during the steady-state at (a) $\mathrm{MI}=0.45$ and $\mathrm{PF}=0.80$. (b) $\mathrm{MI}=0.95$ and $\mathrm{PF}=0.15$.

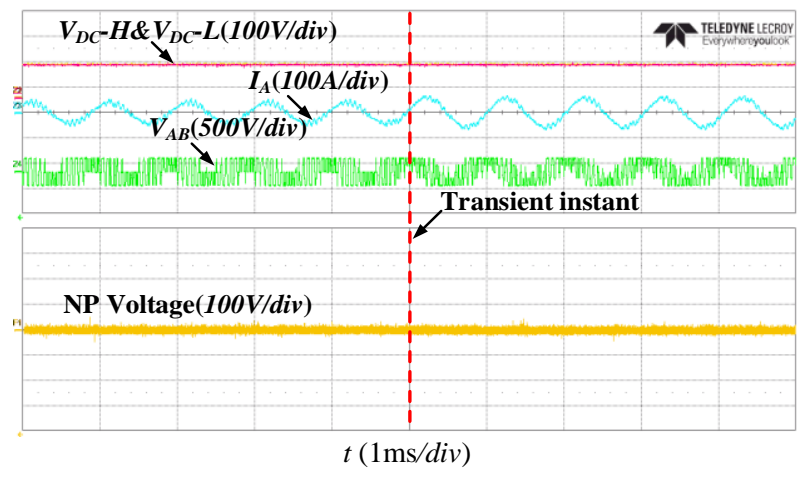

Fig.22. Experimental results of the proposed modulation strategy during the transient-state condition.

takes up a short time compared with the whole period. Fig.21(a) shows the two capacitor voltages, phase current and the line-toline voltage when the $q$-axis current command is $-2 \mathrm{~A}$. At that time, the average modulation index and power factor are 0.45 and 0.80 , respectively. It can be observed that the balanced NP voltage is attained. When the $q$-axis current command is $-15 \mathrm{~A}$, the modulation index of 0.95 and the power factor of 0.15 are the operating condition of the ESG system. As shown in Fig.21(b), the capacitor voltages can also maintain balanced. In addition, the modulation process during transient-state is verified when the modulation index is ramping from 0.6 to 0.9 , suddenly. The results from Fig.22 demonstrate its exceptional dynamic-performance regarding phase current, output voltage and balanced capacitor voltages. The line-to-line voltage, phase current and CMV comparison between the conventional $\mathrm{NTV}^{2}$ method and the proposed strategy is performed when the motor drives operates at $20 \mathrm{krpm}$ in generation mode, as shown in Fig.23. It can be clearly seen that the CMV produced by the presented solution is half of the benchmark method. As the 


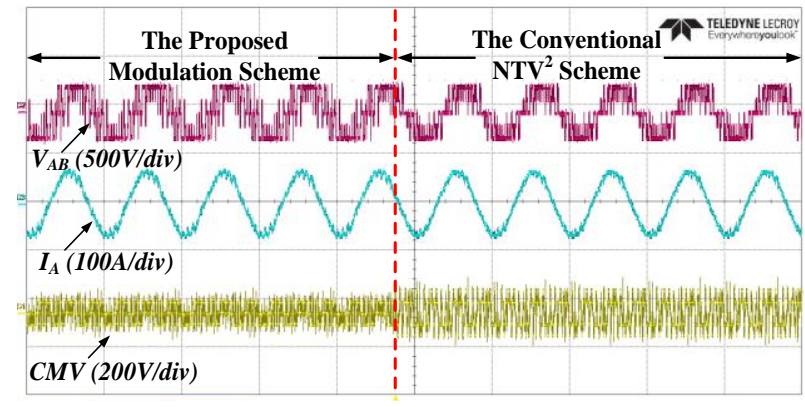

$t(1 \mathrm{~ms} /$ div $)$

Fig.23. Experimental results of the line-to-line voltage, phase current and CMV during modulation strategy transition period.

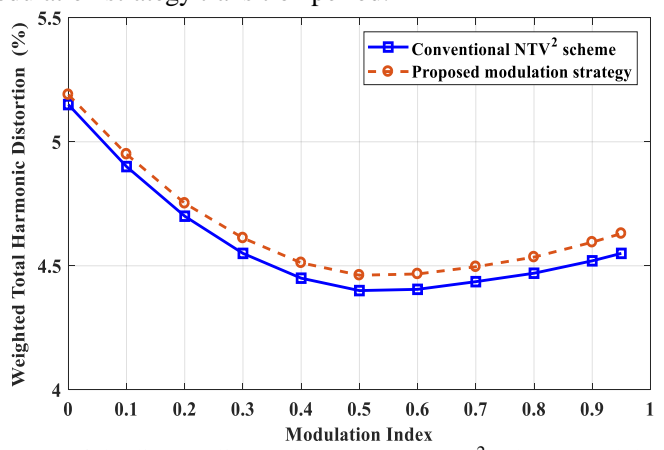

Fig.24. WHTD of the line-to-line voltage under $\mathrm{NTV}^{2}$ scheme and the proposed strategy at different modulation indices.

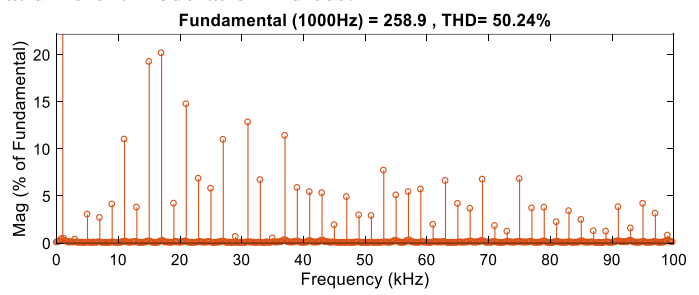

(a)

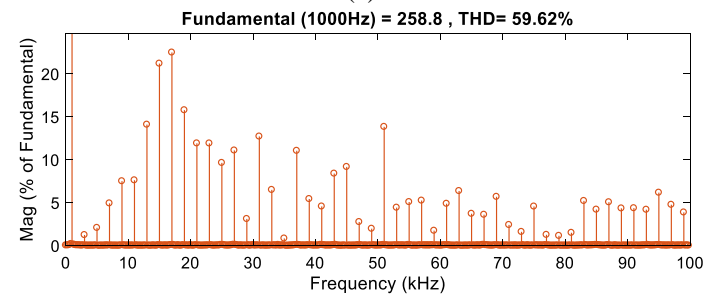

(b)

Fig.25. Harmonic spectra of line-to-line voltage under the steady-state operating point with (a) The conventional $\mathrm{NTV}^{2}$ scheme. (b) The proposed modulation scheme.

selected space vectors are not the nearest three, the THD of phase current of the proposed scheme is $5.87 \%$, which is higher than that of NTV ${ }^{2}$ method with $5.02 \%$.

Fig. 24 shows the weighted THD (WTHD) comparison result of the line-to-line voltage between the benchmark method and the proposed modulation technique under different modulation indices. As shown, when the modulation index is around $0.5 \sim$ 0.6 , WTHD performs the lowest amount since the reference voltage vector is synthesized by $V_{S 1}, V_{S 2}$ and $V_{M 1}$ that are the nearest three virtual space vectors among all applicable vector pairs. Furthermore, it can be seen that both values follow the same trend since two set of strategies deploy the same virtual space vector position and subsector partition. For the proposed scheme, the value is larger than its counterpart, particularly in the higher modulation index region. This is because the three-

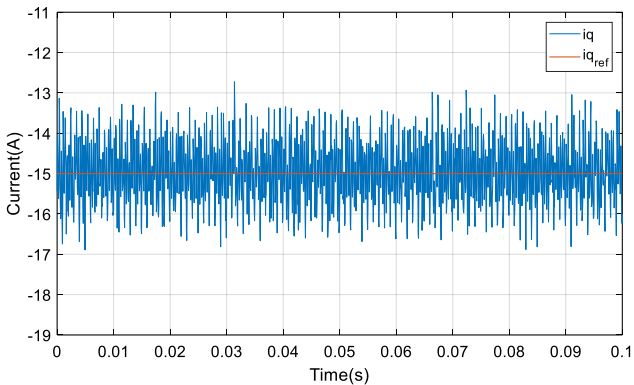

(a)

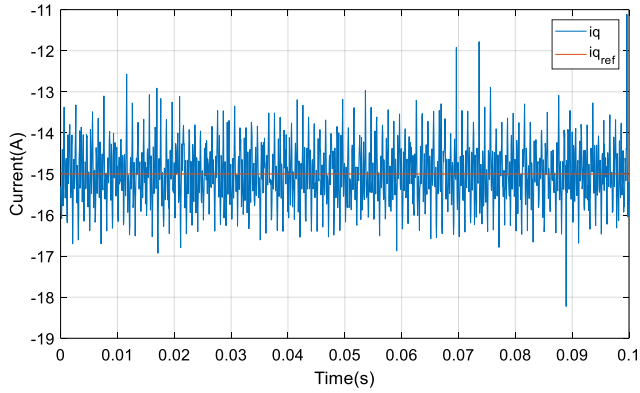

(b)

Fig.26. Quadrature-axis current of applied surface-mounted PMSM in the generation mode with (a) The conventional $\mathrm{NTV}^{2}$ strategy (b) The proposed modulation strategy.

level switching instants occasionally exist in two-phase legs during some scenarios.

Fig.25 presents the harmonic spectra of line-to-line voltage under the conventional $\mathrm{NTV}^{2}$ scheme and the proposed strategy when the ESG system operates in the steady-state operating point. It can be seen that, due to the adopted non-nearest space vectors with lower CMVs, THD of 59.62\% with the introduced method is higher than that of the original scheme. Also, the THD of pole voltage for the prior method and the proposed modulation technique is $68.18 \%$ and $71.32 \%$, respectively.

Since the pole-pair numbers and magnet flux are constant values for our applied surface-mounted PMSM, the torque ripple is proportional to $q$-axis current ripple. In order to evaluate the torque ripple, here, $q$-axis current is measured and displayed by the graph user interface (GUI) when the ESG system operates in power generation mode, in which the $q$-axis reference current is set to be $-15 \mathrm{~A}$ and the $\mathrm{S} / \mathrm{G}$ system delivers approximate active power of $6 \mathrm{~kW}$. It can be observed from Fig.26 that the maximum magnitude of $i_{q}$ spike caused by the presented modulation technique is around $\pm 3.7 \mathrm{~A}$ as compared to $\pm 2.1 \mathrm{~A}$ by its counterpart. Furthermore, the average amount of ripple generated by the proposed strategy $( \pm 1.5 \mathrm{~A})$ roughly increases by $3.1 \%$ compared with the traditional method $( \pm 1 \mathrm{~A})$. Accordingly, the torque ripple would also be proportionally increased in the motor drives.

Additionally, the execution time of the presented approach is tested by the applied controller kit, and the result is $52.7 \mu \mathrm{s}$. Compared with that of the traditional pulse train generation process based on the $\alpha-\beta$ reference frame that costs about $60.6 \mu \mathrm{s}$, it means the amount is approximately reduced by $15 \%$.

\section{CONCLUSION}

An enhanced virtual space vector modulation strategy has been proposed in this paper to realize inherent NP voltage 
balance and CMV reduction for 3L-NPC converters in MEA applications. Despite the fact that conventional $\mathrm{NTV}^{2}$ scheme can address capacitor voltage deviation problem and eliminate undesirable NP voltage ripple for all loads, the CMV magnitude caused by this method is still high for the studied aircraft ESG system. By contrast, lower CMV can be achieved by selecting a series of modified virtual space vectors presented in this work. Furthermore, in order to pursuit high efficiency and simple implementation, the PWM modulator is carried out by a fast calculation approach. The effectiveness and feasibility of the proposed algorithm have been proved by the simulation and experimental results.

Although good modulation performance can be gained, the presented scheme does not have active NP voltage control capability when a disturbance, such as instant voltage droop in the bipolar DC-bus structure ( $\pm 270 \mathrm{VDC})$ of the next-generation MEA, occurs unexpectedly. The improved technique will be discussed in future work.

\section{REFERENCES}

[1] S. Bozhko et al., "Development of aircraft electric starter-generator system based on active rectification technology," IEEE Trans. Transport. Electrific., vol. 4, no. 4, pp. 985-996, Dec. 2018.

[2] G. Buticchi, S. Bozhko, M. Liserre, P. Wheeler and K. Al-Haddad, "OnBoard microgrids for the more electric aircraft-technology review," IEEE Trans. Ind. Electron., vol. 66, no. 7, pp. 5588-5599, Jul. 2019.

[3] F. Gao, S. Bozhko, G. Asher, P. Wheeler and C. Patel, "An improved voltage compensation approach in a droop-controlled DC power system for the more electric aircraft," IEEE Trans. Power Electron., vol. 31, no. 10, pp. 7369-7383, Oct. 2016.

[4] B. Sarlioglu and C. T. Morris, "More electric aircraft: review, challenges, and opportunities for commercial transport aircraft," IEEE Trans. Transport. Electrific., vol. 1, no. 1, pp. 54-64, Jun. 2015.

[5] V. Raveendran, M. Andresen and M. Liserre, "Improving onboard converter reliability for more electric aircraft with lifetime-based control," IEEE Trans. Ind. Electron., vol. 66, no. 7, pp. 5787-5796, Jul. 2019.

[6] R. Teodorescu, F. Blaabjerg, J. K. Pedersen, E. Cengelci and P. N. Enjeti, "Multilevel inverter by cascading industrial VSI," IEEE Trans. Ind. Electron., vol. 49, no. 4, pp. 832-838, Aug. 2002.

[7] M. F. Escalante, J. -. Vannier and A. Arzande, "Flying capacitor multilevel inverters and DTC motor drive applications," IEEE Trans. Ind. Electron., vol. 49, no. 4, pp. 809-815, Aug. 2002.

[8] J. Pou, R. Pindado, D. Boroyevich and P. Rodriguez, "Evaluation of the low-frequency neutral-point voltage oscillations in the three-level inverter," IEEE Trans. Ind. Electron., vol. 52, no. 6, pp. 1582-1588, Dec. 2005.

[9] A. Choudhury, P. Pillay and S. S. Williamson, "DC-Link voltage balancing for a three-level electric vehicle traction inverter using an innovative switching sequence control scheme," IEEE J. Emerg. Sel. Topics Power Electron., vol. 2, no. 2, pp. 296-307, Jun. 2014.

[10] S. Busquets-Monge, J. Bordonau, D. Boroyevich and S. Somavilla, "The nearest three virtual space vector PWM - a modulation for the comprehensive neutral-point balancing in the three-level NPC inverter," IEEE Power Electron. Lett., vol. 2, no. 1, pp. 11-15, Mar. 2004.

[11] F. Guo, T. Yang, C. Li, S. Bozhko and P. Wheeler, "Active modulation strategy for capacitor voltage balancing of three-level neutral-pointclamped converters in high-speed drives," IEEE Trans. Ind. Electron., doi: 10.1109/TIE.2021.3065605

[12] C. Hu et al., "An improved virtual space vector modulation scheme for three-level active neutral-point-clamped inverter," IEEE Trans. Power Electron., vol. 32, no. 10, pp. 7419-7434, Oct. 2017.

[13] X. Wu, G. Tan, Z. Ye, G. Yao, Z. Liu and G. Liu, "Virtual space vector PWM for a three-level neutral-point-clamped inverter with unbalanced DC-links," IEEE Trans. Power Electron., vol. 33, no. 3, pp. 2630-2642, Mar. 2018.

[14] J. Weidong, L. Wang, J. Wang, X. Zhang and P. Wang, "A carrier-based virtual space vector modulation with active neutral-point voltage control for a neutral-point-clamped three-level inverter," IEEE Trans. Ind. Electron., vol. 65, no. 11, pp. 8687-8696, Nov. 2018.
[15] J. M. Erdman, R. J. Kerkman, D. W. Schlegel and G. L. Skibinski, "Effect of PWM inverters on AC motor bearing currents and shaft voltages," IEEE Trans. Ind. Appl., vol. 32, no. 2, pp. 250-259, Mar.-Apr. 1996.

[16] S. Chen, T. A. Lipo and D. Fitzgerald, "Source of induction motor bearing currents caused by PWM inverters," IEEE Trans. Energy Convers., vol. 11, no. 1, pp. 25-32, Mar. 1996

[17] S. Ogasawara, H. Ayano and H. Akagi, "Measurement and reduction of EMI radiated by a PWM inverter-fed AC motor drive system," IEEE Trans. Ind. Appl., vol. 33, no. 4, pp. 1019-1026, Jul.-Aug. 1997.

[18] A. Muetze and C. R. Sullivan, "Simplified design of common-mode chokes for reduction of motor ground currents in inverter drives," IEEE Trans. Ind. Appl., vol. 47, no. 6, pp. 2570-2577, Nov.-Dec. 2011.

[19] H. Akagi, H. Hasegawa and T. Doumoto, "Design and performance of a passive EMI filter for use with a voltage-source PWM inverter having sinusoidal output voltage and zero common-mode voltage," IEEE Trans. Power Electron., vol. 19, no. 4, pp. 1069-1076, Jul. 2004.

[20] D. Han, C. T. Morris and B. Sarlioglu, "Common-mode voltage cancellation in PWM motor drives with balanced inverter topology," IEEE Trans. Ind. Electron., vol. 64, no. 4, pp. 2683-2688, Apr. 2017.

[21] K. Tian, J. Wang, B. Wu, Z. Cheng and N. R. Zargari, "A virtual space vector modulation technique for the reduction of common-mode voltages in both magnitude and third-order component," IEEE Trans. Power Electron., vol. 31, no. 1, pp. 839-848, Jan. 2016.

[22] Haoran Zhang, A. Von Jouanne, Shaoan Dai, A. K. Wallace and Fei Wang, "Multilevel inverter modulation schemes to eliminate commonmode voltages," IEEE Trans. Ind. Appl., vol. 36, no. 6, pp. 1645-1653, Nov.-Dec. 2000.

[23] Huu-Cong Vu, T. D. Nguyen, Tae-Won Chun and H. Lee, "New virtual space vector modulation scheme to eliminate common-mode voltage with balanced neutral-point voltage for three-level NPC Inverters," in Proc. IEEE Energy Convers. Congrs. Expo.-Asia, Jun. 2017, pp. 313-318.

[24] X. Xing, A. Chen, Z. Zhang, J. Chen and C. Zhang, "Model predictive control method to reduce common-mode voltage and balance the neutralpoint voltage in three-level T-type inverter," in Proc. 31st Annu. IEEE Appl. Power Electron. Conf. Expo., Mar. 2016, pp. 3453-3458.

[25] A. Choudhury, P. Pillay and S. S. Williamson, "Modified DC-bus voltage balancing algorithm for a three-level neutral-point-clamped PMSM inverter drive with reduced common-mode voltage," IEEE Trans. Ind. Appl., vol. 52, no. 1, pp. 278-292, Jan.-Feb. 2016.

[26] X. Xing, X. Li, F. Gao, C. Qin and C. Zhang, "Improved space vector modulation technique for neutral-point voltage oscillation and commonmode voltage reduction in three-level inverter," IEEE Trans. Power Electron., vol. 34, no. 9, pp. 8697-8714, Sep. 2019.

[27] W. Jiang et al., "A novel virtual space vector modulation with reduced common-mode voltage and eliminated neutral point voltage oscillation for neutral point clamped three-level inverter," IEEE Trans. Ind. Electron., vol. 67, no. 2, pp. 884-894, Feb. 2020.

[28] X. Xu, Z. Zheng, K. Wang, B. Yang and Y. Li, "A comprehensive study of common mode voltage reduction and neutral point potential balance for a back-to-back three-level NPC converter," IEEE Trans. Power Electron., vol. 35, no. 8, pp. 7910-7920, Aug. 2020.

[29] S. Ohn, J. Yu, R. Burgos, D. Boroyevich and H. Suryanarayana, "Reduced common-mode voltage PWM scheme for full-SiC three-level uninterruptible power supply with small DC-link capacitors," IEEE Trans. Power Electron., vol. 35, no. 8, pp. 8638-8651, Aug. 2020.

[30] F. Guo, T. Yang, S. Bozhko and P. Wheeler, "A novel virtual space vector modulation scheme for three-level NPC power converter with neutralpoint voltage balancing and common-mode voltage reduction for electric starter/generator system in more-electric-aircraft," in Proc. IEEE Energy Convers. Congrs. Expo., Sep. 2019, pp. 1852-1858.

[31] A. Diab, M. Rashed, J. Li, C. Gerada and S. Bozhko, "Performance analysis of PMSM for high-speed starter-generator system," in Proc. IEEE Int. Conf. Elect. Syst. Aircr., Railway, Ship Propuls. Road Vehicles Int. Transp. Electrific. Conf. (ESARS-ITEC), Nov. 2018, pp. 1-7.

[32] A. Tripathi and G. Narayanan, "Analytical evaluation and reduction of torque harmonics in induction motor drives operated at low pulse numbers," IEEE Trans. Ind. Electron., vol. 66, no. 2, pp. 967-976, Feb. 2019.

[33] S. Bozhko et al., "Development of aircraft electric starter-generator system based on active rectification technology," IEEE Trans. Transport. Electrific., vol. 4, no. 4, pp. 985-996, Dec. 2018.

[34] N. B. A and P. Agarwal, "Nearest and non-nearest three vector modulations of NPCI using two-level space vector diagram - a novel approach," IEEE Trans. Ind. Appl., vol. 54, no. 3, pp. 2400-2415, MayJun. 2018 
[35] K. Basu, J. S. S. Prasad, G. Narayanan, H. K. Krishnamurthy and R. Ayyanar, "Reduction of torque ripple in induction motor drives using an advanced hybrid PWM technique," IEEE Trans. Ind. Electron., vol. 57, no. 6, pp. 2085-2091, Jun. 2010.

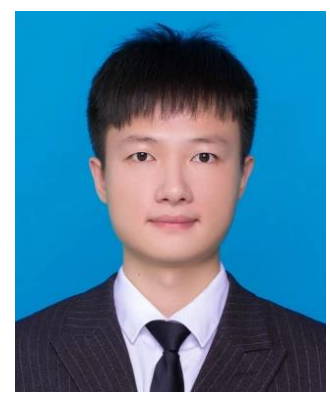

Feng Guo (S'19) received the B.Eng. degree from China University of Mining and Technology, Xuzhou, China, in 2014, and the M.Sc. (Hons.) degree from Northeastern University, Shenyang, China, in 2017, both in Electrical Engineering. Currently, he is working toward the Ph.D. degree with the Power Electronics, Machines and Control Group, University of Nottingham, Nottingham, U.K.

His research interests include pulse-width-modulation strategy, multilevel converters, diagnosis and fault tolerance control, high-speed motor drives and the more-electric-aircraft technologies. From 2016 to 2017, he was a visiting student with The Chinese University of Hong Kong, Shenzhen, China. He was awarded as the Outstanding Graduate Student in 2017.

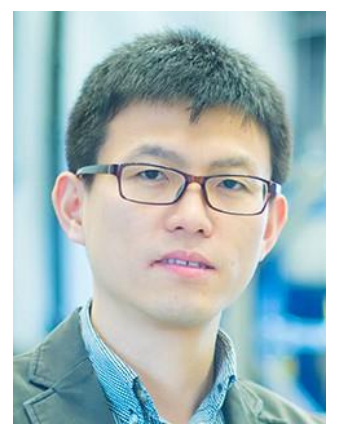

Tao Yang (SM'20) received his MEng Degree from Shanghai Jiao Tong University, China in 2008 and his Ph.D. degree in electrical engineering from the University of Nottingham, UK in 2013.

Since 2013, he has been a Researcher with Power Electronics, Machines and Control Group, University of Nottingham, where he became an Assistant Professor in 2016, and an Associate Professor in 2019. His research interests include high-speed electric motor drive control, power electronic conversion, electrical system design and optimization for more electric/hybrid/all-electric aircraft applications. His $\mathrm{PhD}$ research within EU Clean Sky on "Modelling electrical power system for more-electric aircraft applications" has resulted in him winning the inaugural "Clean Sky Best PhD Award" in 2016.

Dr. Yang is an Associate Editor for the IEEE TRANSACTIONS ON TRANSPORTATION ELECTRIFICATION and Chinese Journal of Aeronautics.

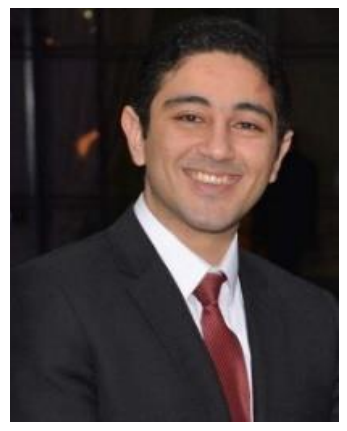

Ahmed M. Diab (S'21) received the B.Sc. (Hons.) and M.Sc. degrees from Zagazig University, Zagazig, Egypt in 2011 and 2016, respectively. He is currently working toward the Ph.D. degree at the University of Nottingham Ningbo China and Institute for Aerospace technology (IAT), university of Nottingham, Nottingham, UK. From 2011 to 2016, he was a research and Teaching Assistant with the Department of Electrical Power and Machines, Zagazig University. His research interests include modeling, control, and stability in high-speed motor/generator systems for transportation electrification applications and in variable-speed wind generators.

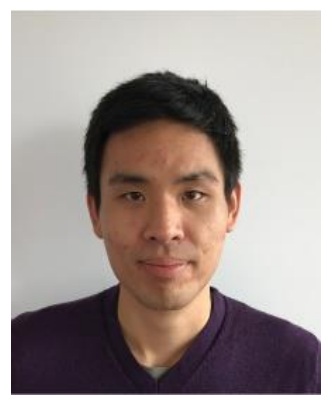

Seang Shen Yeoh received his MSc degree in power electronics (Distinction) and Ph.D degree in electrical engineering from the University of Nottingham, United Kingdom, in 2011 and 2016 respectively. Since then, he has been a research fellow in the same university under the Power Electronics, Machines, and Controls Research Group. His current research interests are in the area of aircraft power systems, namely modelling and stability studies of complex power systems, and new control strategies for highspeed drive systems.

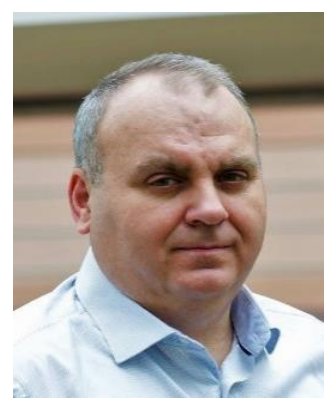

Serhiy Bozhko (M'97-SM'18) received his M.Sc. and Ph.D. degrees in electromechanical systems from the National Technical University of Ukraine, Kyiv City, Ukraine, in 1987 and 1994, respectively. Since 2000, he has been with the Power Electronics, Machines and Controls Research Group of the University of Nottingham, United Kingdom, where currently he is Professor of Aircraft Electric Power Systems and Director of the Institute for Aerospace Technology. He is leading several EU- and industry funded projects in the area of aircraft electric power systems, including power generation, distribution and conversion, power quality, control and stability issues, power management and optimization, as well as advanced modelling and simulations methods.

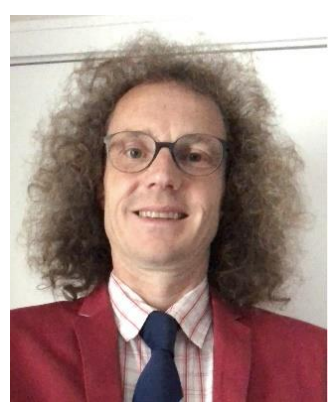

Patrick Wheeler (M'99-SM'11F'21) received his BEng (Hons.) degree in 1990 from the University of Bristol, UK. He received his $\mathrm{PhD}$ degree in Electrical Engineering for his work on Matrix Converters from the University of Bristol, UK in 1994. In 1993 he moved to the University of Nottingham and worked as a research assistant in the Department of Electrical and Electronic Engineering. In 1996 he became a Lecturer in the Power Electronics, Machines and Control Group at the University of Nottingham, UK. Since January 2008 he has been a Full Professor in the same research group.

He was Head of the Department of Electrical and Electronic Engineering at the University of Nottingham from 2015 to 2018. He is currently the Head of the Power Electronics, Machines and Control Research Group, Global Director of the University of Nottingham's Institute of Aerosapce Technology and was the Li Dak Sum Chair Professor in Electrical and Aerospace Engineering. He is a member of the IEEE PELs AdCom and is currently an IEEE PELS Vice-President. He has published over 750 academic publications in leading international conferences and journals. 